\title{
BIODISPONIBILIDADE DE ZINCO DA DIETA DOS PRÉ- ESCOLARES E O EFEITO DA ADIÇÃO DA FARINHA DE BANANA (Musa paradisiaca). ESTUDO EM RATOS.
}

\author{
Alba Luz VÁSQUEZ ${ }^{1}$, Lucia Kiyoko Ozaki YUYAMA ${ }^{2}$, Jaime Paiva Lopes \\ AGUIAR $^{3}$, Risonilce Fernandes Silva SOUZA ${ }^{3}$.
}

RESUMO - Foi avaliada a biodisponibilidade de zinco e o efeito da adição da farinha de banana, em dieta de pré-escolares de uma creche de Manaus, pelo método de depleção e repleção, em ratos. As refeições servidas aos pré-escolares foram coletadas por meio da porção em duplicata, por um período de 18 dias, secas e analisadas quanto a composição centesimal, fibra alimentar, zinco e fítato. O experimento constou de uma fase de depleção de zinco dos ratos, por um período de 35 dias após o nascimento, onde os mesmos receberam ração à base de caseína sem a adição de zinco na mistura salina. A segunda fase foi de 28 dias e o delineamento experimental foi inteiramente casualizado, consistindo de 4 grupos (Dieta dos pré-escolares, dieta dos pré-escolares adicionada da farinha de banana, ração controle à base de caseina, e ração controle à base de caseina, sem a adição de zinco na mistura salina), com 8 animais cada. De acordo com os resultados obtidos, verificou-se baixo teor de zinco na dieta dos pré-escolares. Os animais dos grupos que receberam a dieta dos pré-escolares adicionada ou não de farinha de banana tiveram menor índice de absorção aparente de zinco e menor retenção de zinco nos fềmures quando comparados com o grupo controle $(\mathrm{p}<0,05)$, demonstrando a baixa biodisponibilidade de zinco da dieta, possivelmente, pelos fatores interferentes presentes na mesma. Assim, a farinha de banana na concentração utilizada, não apresentou nenhum efeito na biodisponibilidade de zinco.

Palavras-chave: zinco, dieta de pré-escolares, biodisponibilidade, banana.

Zinc Bioavalability in Pre-school Diets and the Effect of the Addition of the Banana Flour. Study in Rats.

ABSTRACT - It was evaluated zinc bioavailability and the effect of the addition of banana flour to the diet of preschool of Manaus, for the methods of depletion and repletion in rats. The meals served to the preschool ones had been collected by means of the portion in duplicate, for a period of 18 days, dried and analysed for the centesimal composition, alimentary fiber, zinc and phytate. The experiment consisted of a phase of depletion of zinc in the rats, for a period of 35 days after birth, where the same ones had received ration to the base from casein without the addition from zinc in the saline mixture. The second phase was of 28 days and the experimental design was randomized entirely, consisting of 4 groups (Diet of the preschool, added of the banana flour, ration controls the base of casein and ration controls the base of casein, without the zinc addition in the saline mixture), with 8 animals each. According to the results, low concentration of zinc in the diet preschool were detected. The animals of the groups offered ration added with the preschool meals with or without banana flour had smaller index of apparent absorption, smaller zinc clamping in femur, when compared with the controls $(p<0,05)$, demonstrating the low zinc bioavailability of the diet, possibly, due to some influence of the factors present in it. Therefore, banana flour in the concentration used in the present study, did not shown any effect in the zinc bioavailability.

Key-words: zinc, diet of preschool, bioavailability, banana flour.

${ }^{1}$ Pós-graduanda, Ciência de Alimentos, área de concentração em Nutrição - UA. Alexandre Amorin 330, Manaus-AM. CEP 69010-300.

${ }^{2} \mathrm{INPA} / \mathrm{CPCS}$. Orientador, Lab. de Nutrição e Físico-Quimica de Alimentos. Av. André Araújo 1756, Aleixo, Manaus-AM. CEP 69083-000.

${ }^{3}$ INPA/CPCS. Lab. de Nutrição e Físico-Quimica de Alimentos. Av. André Araújo 1756, Aleixo,

Manaus-AM. CEP 69083-000. 


\section{INTRODUÇÃO}

Com o avanço tecnológico e metodológico, tornou-se possível e prático a quantificação dos elementos minerais traços. Dentre estes, o zinco, tem sido reconhecido como nutriente essencial desde 1909 (Tood et al., 1934) e recentemente a sua essencialidade está centrada na transcrição e translação de polinucleotideos e consequentemente no processo da expressão gênica, sendo portanto, fundamental para todas as formas de vida (OMS, 1998). Sua função reguladora, estrutural e catalítica em mais de 200 enzimas o coloca em um patamar de prioridade, pois, a deficiência concorre para o retardo do crescimento, hiperceratose e paraceratose de pele e esofago, assim como, atrofia do timo e gonadas (Sandstead, 1994).

Atualmente, com o desenvolvimento de novas técnicas no campo analítico e instrumental é possivel medir com uma precisão, os teores dos nutrientes contidos nos alimentos. Contudo, somente a análise química não é suficiente para uma caracterização completa do alimento em estudo sob o ponto de vista nutricional, isto porque somente uma parte dos nutrientes contidos nos alimentos se torna biodisponivel ao organismo, ou seja a proporção do que foi consumido que pode ser absorvida e utilizada. Entende-se por utilização o processo intermediário de transporte, armazenamento, assimilação celular e conversão para a forma biologicamente ativa (O'Dell, 1985). Entretanto, de acordo com Cozzolino (1997), há uma forte tendência, para a redefinição de biodisponibilidade como sendo a fração de qualquer nutriente ingerido e absorvido que tem o potencial para suprir as demandas fisiológicas em tecidos alvos.

A absorção de zinco é dependente da concentração, e, ocorre por meio de todo o intestino delgado, principalmente no jejuno. Muitos fatores da dieta foram identificados a partir de estudos experimentais como promotores ou antagonistas potenciais da absorção de zinco. Dentre eles fitato (O'Dell, 1985), cálcio na presença de fitato e fibra (Torre et al., 1991) e interação minerais-minerais como ferro e zinco (Pedrosa \& Cozzolino, 1993, Solomons, 1982), antagonistas potenciais da absorção de zinco. Por outro lado, congêneres de vinho tinto (McDonald \& Margen, 1980), histidina (Scholmerich et al., 1987), proteinas de alto valor biológico, como a proteína animal (Frohlich \& Sandstrom, 1983, Yuyama \& Cozzolino, 1994), frutose (Hoolbrook et al., 1969) e glicose (Turnubull et al., 1990), podem facilitar a absorção e bioconversão.

Apesar da pouca evidência epidemiológica quanto a deficiência de zinco, acredita-se que a mesma esteja amplamente difundida no mundo, e nas crianças de baixo nível sócio-econômico, refletindo no retardo do crescimento (Ruz et al., 1997), e no desenvolvimento cognitivo e motor (Sandstead et al., 1998).

Estudos envolvendo dietas regionais, tem revelado deficiência na oferta energética (Cardoso et al., 1996, Alencar et al., 1997, Nagahama et al., 1998, Yuyama. et al., 1998). Paradoxalmente, a região Amazônica, possui uma variedade de frutos altamente energéticos, dentre eles a banana (Aguiar, 1996). É um fruto amplamente consumido pelas populações de baixa renda, na forma 
in natura, cozida e farinha. A farinha de banana, apresenta atualmente grande potencial industrial para alimentos infantis, produtos dietéticos e de panificação (Banco da Amazônia, 1995). Considerando a farinha de banana como parte integrante do mingau dos pré-escolares e a limitação dos estudos envolvendo biodisponibilidade de zinco em dietas, avaliou-se a biodisponibilidade de zinco da dieta dos pré-escolares e o efeito da adição da farinha de banana, utilizando o rato como modelo experimental.

\section{MATERIAL E MÉTODOS}

A fim de minimizar a contaminação por metais, toda a vidraria foi mantida em banho de ácido nítrico a $30 \%$ por no mínimo 12 horas, sendo em seguida enxaguada com água desionizada por no mínimo 6 vezes, secas em estufa com temperatura controlada e devidamente acondicionada, até o momento das análises. Todos os recipientes, á base de aço inoxidável, utilizados durante a execução do experimento, envolvendo a coleta de dietas, processamento e análises, como: bandejas para secagem das dietas, acessórios para pulverização, peneira, comedouro e bicos dos bebedouros, foram imersos em solução de EDTA $1 \%$ (ácido etilenodiaminotetraacéticoEDTA) por no mínimo 2 horas e enxaguados por no mínimo 6 vezes com água desionizada, secos em estufa com temperatura controlada e acondicionados até o uso.

Foram utilizadas 16 ratas pós- parto (Rattus novergicus, variedade albinus, Rodentia: Mammalia), com 8 filhotes machos cada, provenientes do biotério do Instituto Nacional de Pesquisas da Amazônia (INPA).

As rações à base de caseína foram elaboradas de acordo com Reeves et al (1993), sendo que para um dos grupos controle á base de caseína, não foi adicionado zinco na mistura salina.

As dietas dos pré-escolares foram coletadas por meio da porção em duplicata por um periodo de 18 dias consecutivos na Fundação Hospitais e Creches do Amazonas (HOCRE) e processadas no Laboratório de Nutrição e Físico-quimica de Alimentos do INPA.

A farinha de banana, foi elaborada simulando os hábitos regionais, ou seja, a banana foi descascada, cortadas em rodelas e secas em estufa com circulação de ar forçada a $60^{\circ} \mathrm{C}$, pulverizadas em moinho com facas de aço inoxidável, homogeneizada e adicionada na dieta na proporção de $20 \%$ (20g de farinha de banana em $100 \mathrm{~g}$ de dieta).

O experimento consistiu de uma fase de depleção de zinco de 35 dias após o nascimento dos ratos, ou seja lactação +14 dias, onde as ratas receberam ração, sem adição de zinco na mistura, e, os filhotes por mais 14 dias. Os animais foram mantidos em gaiolas de polietileno com tampa de aço inoxidável com temperatura controlada e constante e ciclo de luz de 12 horas. Todo o material utilizado foi desmineralizado.

O período de repleção foi de 28 
dias e o delineamento experimental foi inteiramente casualizado, constituído de 4 grupos de 8 animais cada, ou seja dois grupos experimentais, onde os animais receberam a dieta dos pré-escolares e dieta dos pré-escolares adicionada de farinha de banana; um grupo controle à base de caseína e um grupo onde os animais receberam ração à base de caseina, sem a adição de zinco na mistura salina. Os animais foram mantidos em gaiolas individuais de aço inoxidável com temperatura controlada e constante e ciclo de luz de 12 horas, orde receberam rações e água ad libitum.

$\mathrm{O}$ crescimento dos animais foi acompanhado por meio de pesagem semanais, durante todo o periodo experimental. O consumo das rações, troca de água dos bebedouros, foram a cada 2 dias. A coleta das fezes foi feita na última semana do experimento. Ao final de cada etapa do experimento os animais foram sacrificados após anestesia com éter etílico para a obtenção do material biológico.

Foram analisadas a composição centesimal da dieta, rações e farinha de banana, seguindo-se as recomendações da (AOAC, 1995) e a fração fibra alimentar, pelo método enzímico-gravimétrico, segundo (Asp et al., 1983). A fração nifext foi determinada por diferença, após a determinação das frações anteriores e o fitato pelo método colorimétrico segundo (Thompson \& Erdman, 1982), modificado por (Lajolo et al., 1991). As determinações dos teores de zinco na dieta, rações, fêmures e testículos foram realizadas segundo o método do Instituto Adolfo Lutz (1985) e de acordo com o Manual da
Perkin Elmer (1967), por leitura direta nas amostras diluídas após digestão via úmida $\left(\mathrm{HNO}_{3} / \mathrm{H}_{2} \mathrm{O}_{2}\right)$. em espectrofotometro de absorção atômica. Utilizou-se o material certificado BOVINE LIVER (NIST-SRM 1577), segundo Cornelis (1992) e Delves (1992).

O teor de zinco no plasma foi determinado por leitura direta das amostras diluidas na proporção de 1:4, em espectrofotômetro de absorção atômica, segundo Rodrigues et al (1989).

Para a avaliação da biodisponibilidade de zinco foram determinados os seguintes parâmetros: indice de crescimento, indice de absorção aparente, onde se relacionou o consumo de zinco e zinco excretado nas fezes pelos animais, assim como zinco fixado nos tecidos (fêmures, testículos e plasma).

Para as análises dos resultados utilizou-se a análise de variância e para efeito de comparação entre as médias dos tratamentos, o teste de Tukey com 5\% de probabilidade (Pimentel Gomez, 1987).

\section{RESULTADOS E DISCUSSÃO}

Verificou-se que as rações apresentaram-se praticamente isocalóricas e isoprotéicas, exceto a ração à base da dieta dos pré-escolares adicionada de farinha de banana (Tab. 1). Em relação ao teor de lipídios encontrou-se variações entre as rações de acordo com a (Tab. 1). O mesmo é extensivo à fração livre de nitrogênio (nifext), sendo observada a maior concentração na ração à base de caseína sem a adição de zinco na mistura salina, e, menor na ração à 
base da dieta dos pré-escolares com adição de farinha de banana. A dieta dos pré-escolares caracterizou-se pela baixa concentração de fibra alimentar, sendo elevada com a adição da farinha de banana (Tab. 1). O teor de zinco foi maior na ração controle $(59,7 \mu \mathrm{g} \%)$, seguido da ração deficiente $(39,3 \mu \mathrm{g} \%)$ e menor nas rações à base da dieta dos pré-escolares $(26,3 \mu \mathrm{g} \%)$ e da dieta dos pré-escolares adicionada de farinha de banana $(23,3$ $\mathrm{mg} \%$ ) (Tab. 1), representando estes valores, aproximadamente $44 \%$ da concen-tração de zinco em relação ao grupo controle. Por outro lado, a concentração significativa de zinco na ração à base de caseína, sem a adição de zinco na mistura salina, justifica-se pelo fato da não remoção do zinco da caseina com EDTA, diferindo dos trabalhos de (Cozzolino, 1992, Mazza, 1992). Cossack \& Hamer (1987) avaliaram a dieta lavada com EDTA utilizada na produção de deficiência de zinco no homem, e, verificaram que o modelo não pode ser utilizado para induzir a deficiência de zinco por um longo período, uma vez que a palatabilidade do produto lavado é muito pobre. Assim, a fim de não interferir na aceitabilidade da ração e desta forma nos resultados, optou-se por não lavar a caseina com EDTA.

A relação molar fitato:zinco encontrou-se acima dos valores críticos tanto nas rações à base da dieta dos pré-escolares quanto na farinha de banana, pelo qual teria uma provável interferência na biodisponibilidade de zinco. Embora, a relação molar fitatoxcálcio/zinco tenha permanecido dentro dos valores permitidos (Tab. 1).

Para a avaliação da depleção de zinco, vários parâmetros foram utilizados, sendo que o ganho de peso dos animais foi o único que se mostrou sensível, sendo significativamente menor no grupo controle à base de caseína, sem a adição de zinco na mistura salina $(80,3 \pm 4,6 \mathrm{~g})$, quando comparado com os animais do grupo controle $(86,3 \pm 6,1 \mathrm{~g}) \quad(\mathrm{p}<0,05)$. Supõem-se que em função do zinco residual na caseina do grupo sem a adição de zinco na mistura salina, o mesmo pode ter contribuido positivamente no ganho de peso dos animais deste grupo, uma vez que a diferença não foi tão acentuada, quando comparados com outros estudos onde a caseína foi lavada com EDTA (Cozzolino,1992, Mazza, 1992)

Em relação aos teores de zinco no plasma, testículos e fềmures, não houve diferença significativa entre os animais dos grupos controle e grupo sem a adição de zinco na mistura salina, demonstrando que provavelmente, o mecanismo adaptativo do organismo do animal, e, o teor de zinco residual na caseína possam ter interferido positivamente nos parâmetros utilizados.

No período de repleção, observou-se que os animais dos grupos que receberam a dieta dos préescolares adicionada da farinha de banana e os do grupo controle, consumiram mais ração, que os animais do grupo da dieta dos préescolares, $(p<0,05)$ (Tab. 2), possivelmente pela melhora da palatabilidade, em função da adição 
Tabela 1. Composição química percentual das rações e da farinha de banana.

\begin{tabular}{|c|c|c|c|c|c|}
\hline Nutrientes & Dieta $^{1}$ & Controle $^{2}$ & Dieta +F. b & $\begin{array}{l}\text { Farinha } \\
\text { Banana }\end{array}$ & $\begin{array}{c}\text { Deficiente em } \\
\text { zinco }{ }^{4}\end{array}$ \\
\hline Energia (Cal) & $390 \pm 0,5^{\text {(a) }}$ & $390 \pm 0,3^{(a)}$ & $363 \pm 1,0^{(b)}$ & $348 \pm 7,9$ & $390 \pm 0,8^{\text {(a) }}$ \\
\hline Proteina (g) & $16,7 \pm 0,3^{(a, b)}$ & $16,6 \pm 0,3^{(a, b)}$ & $16,7 \pm 0,2^{(a, b)}$ & $3,2 \pm 0,8$ & $17,3 \pm 0,3^{(a)}$ \\
\hline Lipídios (g) & $7,3 \pm 0,1^{\text {(a) }}$ & $7,1 \pm 0,1^{(a)}$ & $5,7 \pm 0,3^{(c)}$ & $0,8 \pm 0,1$ & $6,5 \pm 0,01^{(0)}$ \\
\hline Nifext (g) & $64,4 \pm 0,2^{(0)}$ & $65 \pm 0,3^{(a, b)}$ & $60,9 \pm 0,2^{(c)}$ & $81,2 \pm 0,2$ & $65,5 \pm 0,1^{(a)}$ \\
\hline Fibra (g) & 4,1 & $5,0^{*}$ & 9,92 & 7,3 & $5,0^{*}$ \\
\hline Cinza (g) & $3,6 \pm 0,02^{(a)}$ & $2,5 \pm 0,01^{(\text {c) }}$ & $3,3 \pm 0,1^{(b)}$ & $2,7 \pm 0,1$ & $2,4 \pm 0,01^{(0,0)}$ \\
\hline Umidade (g) & $3,9 \pm 0,1^{\text {(a) }}$ & $3,8 \pm 0,02^{(a, b)}$ & $3,2 \pm 0,1^{(c, d)}$ & $5,7 \pm 0,1$ & $3,3 \pm 0,2^{(c)}$ \\
\hline Z̈nco (mg) & $2,6 \pm 0,2^{(0)}$ & $6,0 \pm 0,5^{(a)}$ & $2,3 \pm 0,1^{(c)}$ & $0,4 \pm 0,1$ & $3,9 \pm 0,1^{\text {(b) }}$ \\
\hline Ferro (mg) & $3,4 \pm 0,2$ & & & & \\
\hline Cálcio (mg) & $114,8 \pm 0,7$ & & & & \\
\hline Fitato (mg) & $219 \pm 26,4$ & & & $237 \pm 1,42$ & \\
\hline Razăo molar fitato/zinco & 47 & & & 29 & \\
\hline $\begin{array}{l}\text { Razáo molar Fitato } x \\
\text { cálcio/ zinco }\end{array}$ & 1,4 & & & & \\
\hline
\end{tabular}

-Quantidade de fibra adicionada na raçăo.

Raçâo à base da dieta dos pré-escolares da HOCRE.

${ }^{2}$ Ração à base de caseina. Elaborada de acordo com Reeves et al (1993).

${ }^{3}$ Raçăo à base da dieta dos pré-escolares da HOCRE, adicionada da farinha de banana.

${ }^{4}$ Raçăo à base de caseína, sem a adiçăo de zinco na mistura salina. Elaborada de acordo com Reeves et al (1993).

As médias de mesmas letras no sentido horizontal, năo diferem entre si a nivel de $(p<0,05)$ de significância, pelo Teste de Tukey.

da farinha de banana. Entretanto, divergências são observadas quando comparadas com a literatura, envolvendo estudos com dietas regionais, onde o consumo foi maior, apesar da limitação de determinados nutrientes (Yuyama \& Cozzolino, 1994).

Não houve diferença significativa $(\mathrm{p}<0,05)$ no ganho de peso dos animais dos grupos controle e dieta dos pré- escolares, apesar da variação de zinco consumido (Tab. 2).

Entretanto, o ganho de peso dos animais que receberam a dieta dos pré-escolares adicionada da farinha de banana, foi menor, provavelmente pela presença de fatores interferentes na biodisponibilidade de zinco, como, taninos, presente na banana verde utilizada (Santos \& Zamboni, 1989). 
Os animais que ingeriram maior quantidade de zinco foram os do grupo controle e menor para os dos grupos da dieta dos pré-escolares e a dieta dos pré-escolares adicionada da farinha de banana (Tab. 2). Por sua vez os animais que tiveram o maior índice de absorção aparente foram os do grupo deficiente, sendo menor para os dos grupos da dieta dos pré-escolares, e dieta dos pré-escolares adicionada da farinha de banana, $(\mathrm{p}<0,05)$ (Tab. 2). Este fato, se deve possivelmente, ao aporte protéico dos grupos controle $\mathrm{e}$ deficiente à base de caseina, serem de alto valor biológico. Por outro lado, a quantidade de fitato (1182 $\mathrm{mg})$, presente na dieta dos pré-escolares da HOCRE, é um antagonista potencial que interfere na biodisponibilidade de zinco, aliado ao baixo teor de zinco da dieta. Fato semelhante foi observado em dietas de outras regiões (Tramonte et al, 1996). Não obstante, contrapondo a esses resultados, Yuyama \& Cozzolino (1994), constataram que a fonte protéica da dieta da região Amazônica, foi de alto valor biológico, oriundo principalmente de peixes, aliado ao baixo conteúdo de fitato. Por outro lado, salienta-se que a quantidade de zinco ingerido varia de acordo com a qualidade dos alimentos ingeridos (Jackson \& Lowe, 1992), e, a absorção depende de uma série de eventos como: a forma química na qual o elemento se encontra no alimento ou dieta, do peso molecular, quantidade ingerida, matriz alimentar e presença de ligantes antagônicos e facilitadores (Cozzolino, 1997). Por outro lado, tem se demonstrado que quando a ingestão de

Tabela 2. Média e desvio padrão (DP) do consumo de ração, ganho de peso, zinco ingerido e indice de absorção aparente (IAAp) dos animais ao final do experimento.

\begin{tabular}{|c|c|c|c|c|}
\hline GRUPOS & $\begin{array}{c}\text { CONSUMO DE } \\
\text { RAÇÄO (g) } \\
\text { Média e DP }\end{array}$ & $\begin{array}{l}\text { GANHO DE } \\
\text { PESO (g) } \\
\text { Média e DP }\end{array}$ & $\begin{array}{l}\text { CONSUMO DE } \\
\text { ZINCO(mg/g) } \\
\text { Média e DP }\end{array}$ & $\begin{array}{c}\text { IAAp (\%) } \\
\text { Média e DP }\end{array}$ \\
\hline${ }^{1}$ Controle & $394,0 \pm 17,0^{(a)}$ & $126,4 \pm 21,6^{(a)}$ & $23,0 \pm 1,1^{(\mathrm{a})}$ & $79,4 \pm 1,7^{(\mathrm{b})}$ \\
\hline $\begin{array}{l}2 \text { Dieta dos pré- } \\
\text { escolares }\end{array}$ & $381,1 \pm 18,8^{(a, b)}$ & $112,2 \pm 10,0^{(a)}$ & $10,0 \pm 0,5^{(\mathrm{c})}$ & $64,5 \pm 4,5^{(\mathrm{c})}$ \\
\hline $\begin{array}{l}{ }^{3} \text { Dieta dos pré- } \\
\text { escolares + F.b. }\end{array}$ & $415,2 \pm 35,5^{\text {(a) }}$ & $107,8 \pm 16,9^{(0)}$ & $9,7 \pm 0,9^{(c)}$ & $60,3 \pm 7,5^{(c)}$ \\
\hline $\begin{array}{l}{ }^{4} \text { Sem zinco na } \\
\text { mistura salina. }\end{array}$ & $378,1 \pm 20,6^{(a, b)}$ & $117,9 \pm 11,5^{(\mathrm{a})}$ & $14,9 \pm 0,9^{(b)}$ & $88,2 \pm 1,4^{(a)}$ \\
\hline
\end{tabular}

1 Ração à base de caseina. Elaborada de acordo com Reeves et al (1993).

2 Ração à base da dieta dos pré-escolares da HOCRE.

${ }^{3}$ Raçăo à base da dieta dos pré-escolares da HOCRE, adicionada da farinha de banana.

${ }^{4}$ Raçăo à base de caseína, sem a adiçáo de zinco na mistura salina. Elaborada de acordo com Reeves et al (1993).

Obs: As médias de mesmas letras no sentido vertical năo diferem entre si a nivel de $(p<0,05)$ de significância, pelo teste de Tukey. 
zinco é insuficiente a absorção aumenta, ou seja o organismo tenta manter sua homeostase (Davis et al., 1998).

$\mathrm{O}$ osso contém uma das maiores concentrações de zinco e parece ser um dos parâmetros sensíveis para a avaliação da biodisponibilidade de zinco. Os animais que receberam a dieta dos pré-escolares e dieta dos préescolares adicionada da farinha de banana, apresentaram a menor concentração de zinco nos fêmures, quando comparados com o grupo controle $(153,5 \pm 8,9 \mu \mathrm{g} / \mathrm{g})(\mathrm{p}<0,05)$, (Tab. 3). Resultados semelhantes foram observados em dietas de outras regiões (Tramonte et al., 1996; Pedrosa \& Cozzolino, 1993), diferindo dos estudos da região Amazônica (Yuyama \& Cozzolino, 1994). Vários fatores podem ter interferido na baixa biodisponibilidade de zinco da dieta dos pré-escolares acrescida ou não da farinha de banana, dentre eles, a fonte protéica, que é basicamente vegetal, a relação molar fitato/zinco $(29,8)$ acima dos valores críticos e o baixo teor de zinco na dieta. Tem sido demonstrado que a fonte protéica pode interferir na baixa biodisponibilidade de zinco (Hardie-Muncy \& Rasmussen, 1979).

Em relação a concentração de zinco nos testículos, observou-se que não houve diferença significativa $(p<0,05)$ entre os animais dos grupos controle e dieta dos pré-escolares e dieta dos pré-escolares adicionada da farinha de banana (Tab. 3), diferindo dos estudos de (Yuyama \& Cozzolino, 1994). Cozzolino (1992), encontrou diferença entre os animais adultos e maduros, mas não no grupo dos animais jovens, deduzindo que os resultados podem variar também não só pela

Tabela 3. Média e desvio padrão (DP) da concentração de zinco nos fêmures, testículos e plasma dos animais dos diferentes grupos ao final do experimento.

\begin{tabular}{lccc}
\hline \multicolumn{1}{c}{ GRUPOS } & $\begin{array}{c}\text { Zn nos fêmures } \\
(\mu \mathrm{g} / \mathrm{g})\end{array}$ & $\begin{array}{c}\mathrm{Zn} \text { nos testículos } \\
(\mu \mathrm{g} / \mathrm{g})\end{array}$ & $\begin{array}{c}\mathrm{Zn} \text { no plasma } \\
(\mu \mathrm{g} / \mathrm{mL})\end{array}$ \\
\hline Controle & $153,5 \pm 8,9^{\text {(b) }}$ & $30,2 \pm 4,32^{\text {(a) }}$ & $2,5 \pm 0,1^{\text {(a) }}$ \\
$\begin{array}{l}\text { Dieta dos pré- } \\
\text { escolares }\end{array}$ & $117,6 \pm 9,7^{(\mathrm{c})}$ & $30,2 \pm 2,51^{\text {(a) }}$ & $2,4 \pm 0,2^{\text {(a) }}$ \\
$\begin{array}{l}\text { Dieta dos pré- } \\
\text { escolares+F.b. }\end{array}$ & $118,1 \pm 5,7^{\text {(c) }}$ & $32,0 \pm 3,71^{\text {(a) }}$ & $2,2 \pm 0,2^{\text {(a) }}$ \\
& & & \\
Deficiente & $176,4 \pm 15,5^{\text {(a) }}$ & $27,8 \pm 2,74^{\text {(a) }}$ & $2,1 \pm 0,3^{\text {(a) }}$ \\
\hline
\end{tabular}

Raçäo à base de caseína. Elaborada de acordo com Reeves et al (1993).

Ração à base da dieta dos pré-escolares da HOCRE.

Ração à base da dieta dos pré-escolares da HOCRE, adicionada da farinha de banana.

Ração à base de caseina, sem a adição de zinco na mistura salina. Elaborad de acordo com Reeves et al (1993). Obs: As médias de mesmas letras no sentido vertical não diferem entre si a nível de $(p<0,05)$ de significância pelo teste de Tukey. 
biodisponibilidade do elemento estudado como pela resposta nas diferentes fases de desenvolvimento. Por outro lado, o diagnóstico da deficiência leve de zinco, é muito difícil de se avaliar por não se dispor no momento, de indices sensiveis do estado deste mineral (Sandstrom, 1991).

Em relaçâo ao teor de zinco no plasma, não observou-se diferença significativa $(p<0,05)$ entre os animais do grupo controle e os do grupo da dieta dos pré-escolares e dieta dos pré-escolares adicionada de farinha de banana (Tab. 3). Este fato corrobora com o reportado pela literatura, da existência de um mecanismo homeostático, presente na deficiência dietética de zinco, demonstrando que o zinco plasmático é um pobre indicador na presença de deficiência leve ou marginal de zinco.

\section{CONCLUSÕES}

Conclui-se que o teor de zinco na dieta dos pré-escolares foi baixo, possivelmente pela alta freqüência de alimentos pobres neste mineral. A biodisponibilidade de zinco da dieta dos pré-escolares, quando avaliada pela retenção de zinco nos fềmures, e indice de absorção aparente de zinco, foi baixa, possivelmente em função dos fatores interferentes presentes na dieta, como a fonte protéica, predominantemente de origem vegetal, alto conteúdo de fitato (hexafosfato de mio-inositol) e baixo teor de zinco. A farinha de banana na concentração utilizada, não apresentou nenhum efeito na biodisponibilidade de zinco.

\section{AGRADECIMENTOS}

A Fundação Hospital e Creches do Amazonas, pela doação das dietas, ao INPA pelo suporte financeiro PPI 53200, aos técnicos Aiub Dantas Atem e Lucimar Siqueira.

\section{Bibliografia Citada}

Aguiar, J.P.L. 1996. Tabela de composição de alimentos da Amazônia. Acta Amazonica, $26(1 / 2): 121-126$.

Alencar, F.H.; Yuyama, L.K.O. 1997. Situação alimentar e mutricional de pré-escolares do Municipio de Barcelos-AM. 1995. In: Congresso de Pediatria. 1997. São Paulo. Arquivos Brasileiros de Pediatria, 4(Suppl.1): p.S145.

Asp. N.G.; Johansson, C.G.; Halmer, H.; Siljestron, M. 1983. Rapid enzimatic assay of insoluble and soluble dietary fiber. J. Agric. Food Chem., 31: 476-478.

A.O.A.C. 1995, Association Official Analytical Chemist. Official methods of analysis. 14 Ed. Arlington, , 1141p.

Banco da Amazônia. 1995. Estudo exploratório da cultura da banana no Brasil e na Região Norte. Estudos setoriais 7. Belém-Pará. $34 \mathrm{p}$.

Cardoso, A.S.; Yuyama, L.K.O.; Nagahama, D. 1996. Avaliação do padrão alimentar de mulheres atendidas em três centros de saúde de Manaus, AM. In:

IV Congresso da Sociedade Brasileira de Alimentação e Nutrição. 1996. São Paulo. Sociedade Brasileira de Alimentação e Nutrição, SBAN. Livro de resumos. São Paulo, p.33.

Cornelis, R. 1992. Use of references materials in trace element analysis of fudstuffs. Food Chem. 43: 307-313.

Cossack, Z.T., Hamer, VanDen, C.J.A. 1987. Evaluation of the EDTA-washed diet for use in the experimental production of zinc deficiency in human subjects. Int. J. Vitam. Nutr. Res. 57:99-102. 
Cozzolino, S.M.F. 1997. Biodisponibilidade de minerais. Rev. Nutr., 10 (2): 87-98.

Cozzolino, S.M.F. 1992, Biodisponibilidade de zinco em dieta. Estudo com ratos em diferentes fases do desenvolvimento. Universidade de São Paulo, Tese LivreDocente. $118 \mathrm{p}$.

Davis, S.R.; Mcmahon, R.J.; Cousins, R.J. 1998. Metallothionein knockout and transgenic mice exhibit altered intestinal processing of zinc with unifor zinc-dependent zinc transporter expression. J. Nutr. $12: 825-831$.

Delves, H.T. 1992, Sample preparation handling. Food Chem. 43: 227-281.

Frohlich, W. \& Sandstrom, B. 1983. Zinc absorption from composite meals. In: Nutritional bioavailability of zinc. Am. Chem. Soc. Symp. Series. (210): 211-221.

Hardie-Muncy, D.A.; Rasmussen, A.I. 1979. Interrelationship between zinc and protein level and source in weanling rats. J. Nutr, 109: $321-329$.

Hoolbrook, J.T.; Smith, J.C.; Reiser, SH. 1969. Dietary fructose or starch: effects on copper, zinc, iron, manganese, calcium and magnesium balances in humans. Am. J. Clin. Nutr., 49: 1290-1294.

IAL. Instituto Adolfo Lutz. 1985. Normas analiticas, métodos químicos e fisicos para análises de alimentos. São Paulo. 1:533p.

Jackson, M.J., Lowe, N.M, 1992. Physiological role de zinc. Food Chem. 43:233-38.

Lajolo, F.M.; Marquez, U.M.L.; FilisettiCozzi,T.M.C.C.; McGregor, I. 1991.Chemical composition and toxic compound in rapeseed (Brasica napus, I.) Cultivars grown in Brazil. J.Agric. Foods Chem., 39(11): 1933-1937.

Manual da Perkin Elmer. 1967. Analytical methods for atomic absorption spectrophotometry. Bc 15 (Manual do Mod 373).

Mazza, R.P.D. 1992. Biodisponibilidade de zinco de diferentes fontes de suplementação em dieta regional de São Paulo. (Estudo em ratos). Universidade de São Paulo, São Paulo. Tese doutorado. 190p.
McDonald, J.T.; Margen, S, 1980. Wine versus ethanol in human nutrition. IV. Zinc balance. Am. J. Clin. Nutr., 33: 1096-1102.

Nagahama, D., Yuyama, L.K.O., Aguiar, J.P.L., Macedo S.H.M., Yonekura, L. 1998. Composição quimica percentual de adequação da alimentação dos servidores do Instituto Nacional de Pesquisas da Amazônia, Manaus, AM. In: XVI Congresso Brasileiro de Ciência e Tecnologia de Alimentos, 1998, Rio de Janeiro. Anais do Congresso Brasileiro de Ciência e Tecnologia de Alimentos: Sociedade Brasileira de Ciência e Tecnologia de Alimentos, SBCTA. p.601.

O'Dell, B.L. 1985. Bioavalability of and interaction among trace elements. In: Chandra, R.K. Traces elements in nutrition of children. New York. Raven Press, p. 41-62.

O.M.S. 1998. Organização Mundial de Saúde. Elementos traço na nutrição e saúde humanas. São Paulo, Roca. 297p.

Pedrosa, L.F.C.; Cozzolino, S.M.F. 1993. Efeito da suplementação com ferro na biodisponibilidade de zinco em uma dieta regional do nordeste do Brasil. Rev. Saúde Públ., 27(4): 266-270.

Pimentel Gomez, F. 1987. Curso de Estatística experimental. 12 ed. Piracicaba. 467p.

Reeves, P.G.; Nielsen. F.H.; Fahey, G. C. 1993. AIN-93 Purified diets for laboratory rodents: Final report of the American Institute of Nutrition Ad Hoc writing Committe on the reformulation of the AIN-76 ${ }^{A}$ rodent diet. J. Nutr. 121: 1939 1951.

Rodrigues, M.P., Narizano, A.; Demezlo, V.; Cid, A.A. 1989. A simple method for a determination of zinc human plasma levels by flame atomic absorption spectrophotometry. At. Spectros., 10 (2); 68-70.

Ruz, M.; Castilo-Duran, C.; Lara, X.; Codoceo, J.; Rebolledo, A.; Atalah, E. 1997. A 14 mo zinc supplementation trial in apparently healthy Chilean preschool children. Am. J. Clin. Nutr. 66: 1406-1413. 
Sandstead, H.H., Penland, J.G., Alcock, N.W., Daval;, H.H., Chen, X.C., Li, J.S., Zhao, F., Yang, J.J. 1998. Effects of repletion with zinc and other micronutrients on neuropsychologic performance and growth of chinese children. Am. J. Clin. Nutr. 68 (Suppl.): 470-475.

Sandstead, H.H. 1994. Understanding zinc: recent observations and interpretations. $J$. Lab. Clin. Med., 124: 322-327.

Sandstrom, B. 1991. Zinc: The functional significance of marginal deficiency. In: PIETRZIK, K. (Ed) Modern lifestyles, lower energy intake and micronutrient status. London: Springer-Verlag, 181-189.

Santos, M.C.; Zamboni, C.Q. 1989. Caracterização de banana (Musa sp.) em produtos industrializados, por coloração diferencial de células de tanino, Ver. Inst. Adolfo Lutz. 49(1): 35-39.

Scholmerich, J.et al... 1987. Bioavailabiliy of zinc from zinc histidine complexes. II. Studies on patients with liver cirrosis and the influence of the time of application. Am. J. Clin. Nutr., 45: 1487-1491.

Solomons, N.W. 1982. Interacciones entre zinc y factores dietéticos. Arch. Latinoam. Nutr. 32(1): 26-31.

Thompson, D.V.; Erdman, J.W.Jr. 1982.Phytic acid determination in soybeans. J. Food Sci. 47: 513-517.

Tood, W.T; Elvehejem, C.A., Hart, E.B. 1934. Zinc the nutrition of the rat. Am. J. Physiol. 107: 146-156.

Torre, M.; Rodriguez, R.; Saura-Calixto F. 1991. Effects of dietary fiber and phytic acid on mineral availability. Crit. Rev.Food Sci.Nutr. 1(1): 1-22.

Tramonte, V.L.C.G.; Santos, S.; Cozzolino,
S.M.F. 1996. Biodisponibilidade de zinco de dieta regional de Santa Catarina. In : Congresso Nacional da SBAN, 2-5, 1996, São Paulo. IV Congresso Nacional da Sociedade Brasileira de Alimentação e Nutrição: Sociedade Brasileira de Alimentação e Nutrição, p. 8.

Turnbull, A. J., Blakeborough, P., Thompson, R.P.H. 1990. The effects of dietary ligants on zinc uptake at the porcine intestinal brush-border membrane. Br. J. Nutr. 64: 733-41.

Yuyama, L.K.; Aguiar, J.P.L.; Macedo, S.H.M; Yonekura, L.; Nagahama, D.; Alencar, F.H. 1998. Perfil nutricional de pré-escolares do Municipio de Nhamundá, AM. In: XVI Congresso Brasileiro de Ciências e Tecnologia de Alimentos, 15-17, 1998, Rio de Janeiro. Anais do Congresso Nacional da Sociedade Brasileira de Alimentação e Nutrição: Sociedade Brasileira de Ciência e Tecnologia de Alimentos, SBCTA. 684-687.

Yuyama, L.K.O.; Cozzolino, S.M.F. 1994. Biodisponibilidade de zinco em dieta regional de Manaus, AM. Estudo em ratos. Acta Amazonica. 24(30): 265-274. 